\title{
HIGH-FREQUENCY MEASUREMENTS OF THE SPECTRUM OF SAGITTARIUS A*
}

\author{
E. Serabyn, ${ }^{1,2}$ J. Carlstrom, ${ }^{3}$ O. Lay, ${ }^{4}$ D. C. Lis, ${ }^{1}$ T. R. Hunter, ${ }^{5}$ J. H. Lacy, ${ }^{2,6}$ and R. E. Hills ${ }^{7}$ \\ Received 1997 July 10; accepted 1997 September 22; published 1997 October 27
}

\begin{abstract}
We report near-simultaneous interferometric measurements of the spectrum of Sagittarius A* over the 5-354 $\mathrm{GHz}$ range and single-dish observations that have yielded the first detection of Sgr A* at $850 \mathrm{GHz}$. We confirm that Sgr A*'s spectrum rises more steeply at short millimeter wavelengths than at centimeter wavelengths, leading to a near-millimeter/submillimeter excess that dominates its luminosity. Below $900 \mathrm{GHz}$, Sgr A*'s observed luminosity is $70 \pm 30 \mathrm{~L}_{\odot}$. A new upper limit to Sgr A*'s $24.3 \mu \mathrm{m}$ flux, together with a compilation of other extant IR data, imply a far-infrared spectral turnover, which can result from either an intrinsic synchrotron cutoff or excess extinction near Sgr A*. If the former applies, Sgr A*'s total synchrotron luminosity is $<10^{3} L_{\odot}$, while in the latter case it is $<3 \times 10^{4} L_{\odot}$ if spherical symmetry also applies.
\end{abstract}

Subject headings: galaxies: nuclei - Galaxy: center

\section{INTRODUCTION AND OBSERVATIONS}

Because of its relatively weak emission, Sgr A*, the compact nonthermal radio source (CNRS) at the center of our Galaxy, remains a difficult nut to crack. Nevertheless, intriguing models based on accretion onto a massive black hole are beginning to emerge (reviewed in Morris \& Serabyn 1996). To date, Sgr A* has been detected almost exclusively at radio through submillimeter wavelengths, where its spectral characteristics resemble those of extragalactic CNRSs (Lo 1994). At centimeter wavelengths, Sgr A*'s flux density, $S_{v}$, is reasonably well described by a weak frequency power law, $S_{v} \propto v^{\alpha}$, with a centimeterwave spectral index of $\alpha_{\mathrm{cm}} \approx 0.2-0.3$, but modest time variability (Zhao et al. 1992, hereafter Zh92) prevents assignment of a definitive spectral index. This simple characterization likely fails at higher frequencies, as near-millimeter (here $\lambda=$ 1-3 mm) data suggest a steeper spectrum (Zylka, Mezger, \& Lesch 1992, hereafter Zy92; Serabyn, Carlstrom, \& Scoville 1992). At submillimeter wavelengths, with measurements only recently reaching $670 \mathrm{GHz}$ (Zylka et al. 1995, hereafter Zy95), the situation is less settled. However, Sgr A*'s generally positive spectral index clearly implies a luminosity dominated by its highest frequency emission, and so to better define this emission we undertook (1) extension of interferometric flux measurements of $\operatorname{Sgr} A^{*}$ to submillimeter wavelengths, (2) inclusion of lower $v$ interferometric measurements to provide a broadband, near-simultaneous spectrum, (3) multiepoch near-millimeter measurements, (4) extension of single-dish measurements to the far-infrared (FIR) atmospheric cutoff, and (5) midinfrared (MIR) measurements just beyond the atmosphere's opaque FIR band.

To cover the full extent of the near-millimeter/submillimeter range currently accessible interferometrically, observations with the Owens Valley Radio Observatory (OVRO) and Caltech Submillimeter Observatory-James Clerk Maxwell Tele-

\footnotetext{
${ }^{1}$ California Institute of Technology, 320-47, Pasadena, CA 91125.

${ }^{2}$ Visiting Astronomer at the Infrared Telescope Facility, which is operated by the University of Hawaii under contract with NASA.

${ }^{3}$ Department of Astronomy and Astrophysics, University of Chicago, Chicago, IL 60637.

${ }^{4}$ NASA/Ames Research Center, Moffett Field, CA 94035.

${ }^{5}$ Smithsonian Astrophysical Observatory, Cambridge, MA 02138.

${ }^{6}$ University of Texas, Austin, TX 78712.

${ }^{7}$ Mullard Radio Astronomy Observatory, Cambridge CB3 OHE, Engand.
}

scope (CSO-JCMT) interferometers were carried out at frequencies ranging from 100 to $354 \mathrm{GHz}(\lambda=3.0-0.85 \mathrm{~mm})$. To minimize possible spectral distortions arising from time variability, the observations were confined to a relatively short (12 day) measurement interval: 1993 March 19-30. While the resultant data are not quite simultaneous, earlier studies suggest somewhat longer $(\approx 1$ month) variability timescales (Zh92; Zy95), allowing a characterization of our interferometric data as "near simultaneous." VLA centimeter-wave monitoring measurements (Zh92; J.-H. Zhao 1997, private communication) falling within our 12 day window extend our spectrum down to $5 \mathrm{GHz}$, yielding a near-simultaneous coverage of close to two decades in frequency $(5-354 \mathrm{GHz}$; Fig. 1), a span similar to that of Wright \& Backer (1993) but at frequencies about fourfold higher. The highest frequency multibaseline interferometric images obtained are those from OVRO, which now show a single, unresolved point source at the position of Sgr $\mathrm{A}^{*}$, yielding an upper limit to the $230 \mathrm{GHz}$ source diameter of $1^{\prime \prime}$.

Additional measurements outside our 12 day window extend our spectral coverage, at the cost of giving up the near-simultaneity constraint. A $1.5 \mathrm{GHz}$ VLA measurement from 1993 February (Zh92; J.-H. Zhao 1997, private communication) extends our spectrum to lower $v$, while single-dish upper limits at $\lambda=350$ and $24.3 \mu \mathrm{m}$, obtained with the CSO bolometer in 1993 May and the spectrometer Irshell at the Infrared Telescope Facility in 1993 June constrain higher $v$ emission. Combining all of these data yields a spring 1993 spectrum with constraints over four decades in frequency, from $\lambda=20 \mathrm{~cm}$ to nearly 20 $\mu \mathrm{m}$ (Fig. 1).

To assess the possible effects of time variability, additional near-millimeter interferometry was carried out with CSOJCMT during 1995 October 23-28 and with OVRO during 1996 February 6-15 (Fig. 2). Each of these intervals was again short enough to be characterized as near simultaneous.

Finally, on 1996 April 28, single-dish observations at $\lambda=$ $350 \mu \mathrm{m}(850 \mathrm{GHz})$ were undertaken with the CSO's new submillimeter camera SHARC (Wang et al. 1996), resulting in the first detection of $\mathrm{Sgr} \mathrm{A}^{*}$ at this wavelength (Fig. 3 [Pl. L4]). At $350 \mu \mathrm{m}, \operatorname{Sgr} \mathrm{A}^{*}$ is quite weak relative to the surrounding large-scale dust emission features, but a peak is clearly present at the correct relative location. Strip scans through Sgr A* suggest a flux of $\sim 5 \mathrm{Jy}$, while point-source subtraction yields 


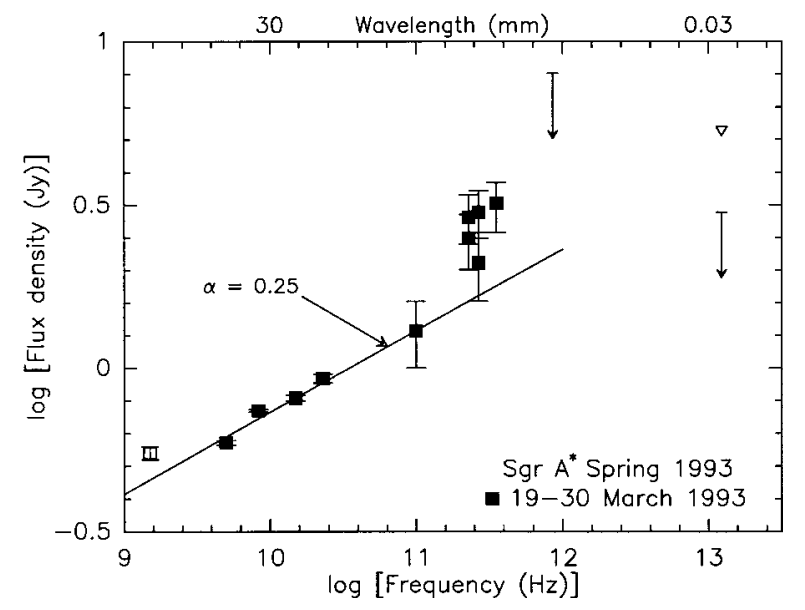

FIG. 1.-Spring 1993 spectrum of Sgr A*. The filled squares are all from the 1993 March 19-30 interval and comprise our near-simultaneous "Mar 1993" spectrum. The remainder of the points fall outside this time frame: the open square at $1.5 \mathrm{GHz}$ is from 1993 February 8 , the $350 \mu \mathrm{m}$ upper limit is from 1993 May 8 , and the $24.3 \mu \mathrm{m}$ upper limit is from 1993 June 3. The triangle corresponds to our $24.3 \mu \mathrm{m}$ limit corrected for interstellar extinction, $5.4 \mathrm{Jy}$. The line shows the best-fit power law $\left(\alpha_{\mathrm{cm}}=0.25\right)$ to the measurements between 5 and $100 \mathrm{GHz}$.

$\sim 9 \mathrm{Jy}$, so we adopt $7 \pm 2 \mathrm{Jy}$ (the map rms noise is also $2 \mathrm{Jy}$ ) as the best estimate for Sgr A*'s $850 \mathrm{GHz}$ flux (Fig. 2). Calibration of our data was with respect to NRAO 530 and Neptune for the interferometry, Uranus for the SHARC map, and Sgr A West IRS1 for the Irshell data.

\section{RESULTS}

Our March 1993 data show a 5-100 GHz spectrum that is very well described by the best-fit power law $S_{v}=$ $\left(v_{\mathrm{GHz}} / 35\right)^{0.25}$ (Fig. 1). However, the higher $v$ fluxes (230-354 $\mathrm{GHz}$ ) exceed this power law by about 1-2 Jy, or roughly a factor of 2 . The observed $1.3 \mathrm{~mm}$ flux of $\approx 3 \mathrm{Jy}$ is consistent with our earlier interferometry (Serabyn et al. 1992) and with single-dish continuum measurements (Zy92; Dent et al. 1993; Zy95). Between 100 and $230 \mathrm{GHz}$ (i.e., near $\lambda=2 \mathrm{~mm}$ ), our March 1993 spectrum yields a spectral index, $\alpha_{2 \mathrm{~mm}}$, of 0.88 , also consistent with earlier results (0.9; Serabyn et al. 1992). (Of course, an even larger intrinsic $\alpha$ could apply over a more limited frequency range.) Given our short measurement interval, the spectral steepening near $2 \mathrm{~mm}$ and the concommitant near-millimeter flux excess are likely not a result of source variability, but to further allay doubts in this regard we also examined the centimeter-wave measurements (Zh92) just beyond our 12 day window. As slightly flatter centimeter-wave spectra were present 1 and 2 weeks prior to our observations, allowing for centimeter-wave variability would thus only exacerbate the near-millimeter excess.

Sgr A*'s near-millimeter spectral index can be explored further via our 1995/1996 data. At near-millimeter wavelengths, Sgr A* was $\approx 25 \%$ brighter in both 1995 October and 1996 February than in 1993 March (Fig. 2). At $\lambda>1 \mathrm{~mm}$, an increased flux is seen at all $\lambda$ values measured, and the spectral shape is preserved (this may not hold at $\lambda<1 \mathrm{~mm}$, but these short $\lambda$ values may be more degraded by the atmosphere). In particular, $\alpha_{2 \mathrm{~mm}}$ is unaltered, being 0.86 in 1996 February. The 1995 October $1.3 \mathrm{~mm}$ flux is also consistent with the 1996 February spectrum, which suggests that slow, low-amplitude drifts (timescale of $\sim 1 \mathrm{yr}$ ) dominate Sgr A*'s near-millimeter

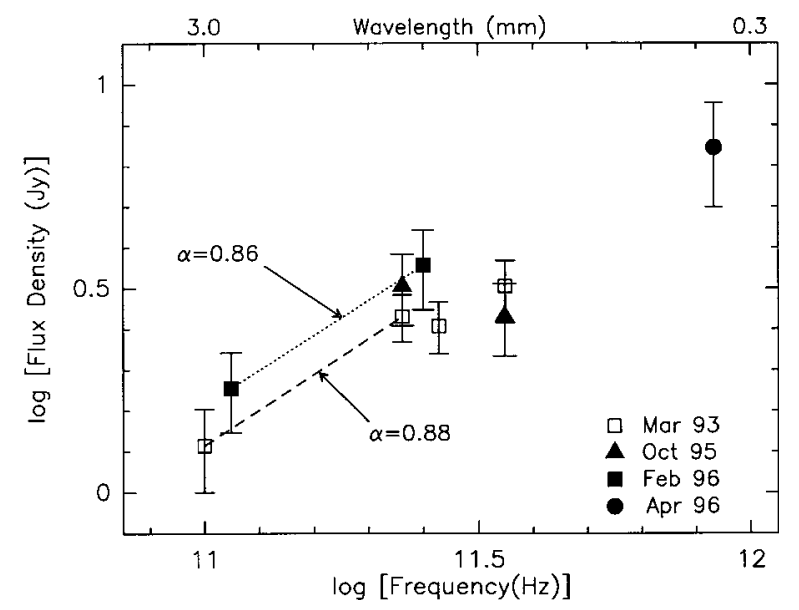

FIG. 2.-Comparison of the near-millimeter/submillimeter 1993 and 1995/ 1996 spectra of Sgr A*. The 1993 data from Fig. 1 at similar frequencies have been averaged for this plot. The spectral indices given are for the two lowest frequency near-simultaneous data points at each epoch. Also included is the detection of Sgr A* at $350 \mu \mathrm{m}$.

variability. Thus, with several epochs of near-millimeter interferometry all consistently showing $\alpha_{2 \mathrm{~mm}} \approx 0.9$, so that $\alpha_{2 \mathrm{~mm}} \gtrsim 3 \alpha_{\mathrm{cm}}$, it is clear that Sgr A*'s spectrum rises much more steeply near $\lambda=2 \mathrm{~mm}$ than at centimeter wavelengths, a conclusion also supported by recent observations of Falcke (1997). A common spectral index for the entire centimeter/ millimeter/submillimeter range thus receives little observational support, even in the time-averaged sense suggested by Duschl \& Lesch (1994).

Limited data at $\nu>300 \mathrm{GHz}$ provide only a restricted view of Sgr A*'s submillimeter spectrum (see also Zy95), but Figure 2 suggests a continued rise to our atmosphere's FIR cutoff, with perhaps some flattening in between. Our 1996 data (February through April) are consistent with a submillimeter spectral index, $\alpha_{\text {smm }}$, of $0.5 \pm 0.45$ (between 251 and $860 \mathrm{GHz}$ ), thus allowing for a submillimeter spectrum ranging from flat to a continued rise at the $\alpha \approx 0.9$ near-millimeter rate. However, a more steeply rising near-millimeter/submillimeter spectrum, as originally proposed by $\mathrm{Zy} 92$, is ruled out.

Given the uncertainty in $\alpha_{\text {smm }}$ and the $\sim 25 \%$ flux changes evident in Figure 2, it is impossible to assign a unique value to Sgr A*'s integrated source luminosity to the FIR atmospheric cutoff. However, consideration of various limiting cases indicates that Sgr A*'s integrated luminosity for $\nu<900 \mathrm{GHz}$ is $\approx 70 \pm 30 L_{\odot}$, of which only $\approx 3 L_{\odot}$, or $4 \%$, emerges in the radio-millimeter power-law component. Even $70 L_{\odot}$ is almost certainly a lower bound to Sgr A*'s luminosity, as its emission likely extends to yet higher $\nu$. However, Sgr A*'s unobserved FIR emission is tightly constrained by our 350 and $24 \mu \mathrm{m}$ data (Figs. 1 and 2). As these $\lambda$ values closely constrict the terrestrial atmosphere's opaque FIR band, they largely rule out a significant FIR flux rise for Sgr $A^{*}(\S 3)$. Instead, our extinctioncorrected $24.3 \mu \mathrm{m}$ limit, together with additional MIR and nearinfrared (NIR) limits (Fig. 4), instead imply a spectral turnover in the $1-10 \mathrm{THz}$ range (see also $\mathrm{Zy} 95$ ).

\section{DISCUSSION}

Two components are thus required to describe Sgr A*'s spectrum: a synchrotron power-law component at radio frequencies and a near-millimeter/submillimeter excess. Dust models for 


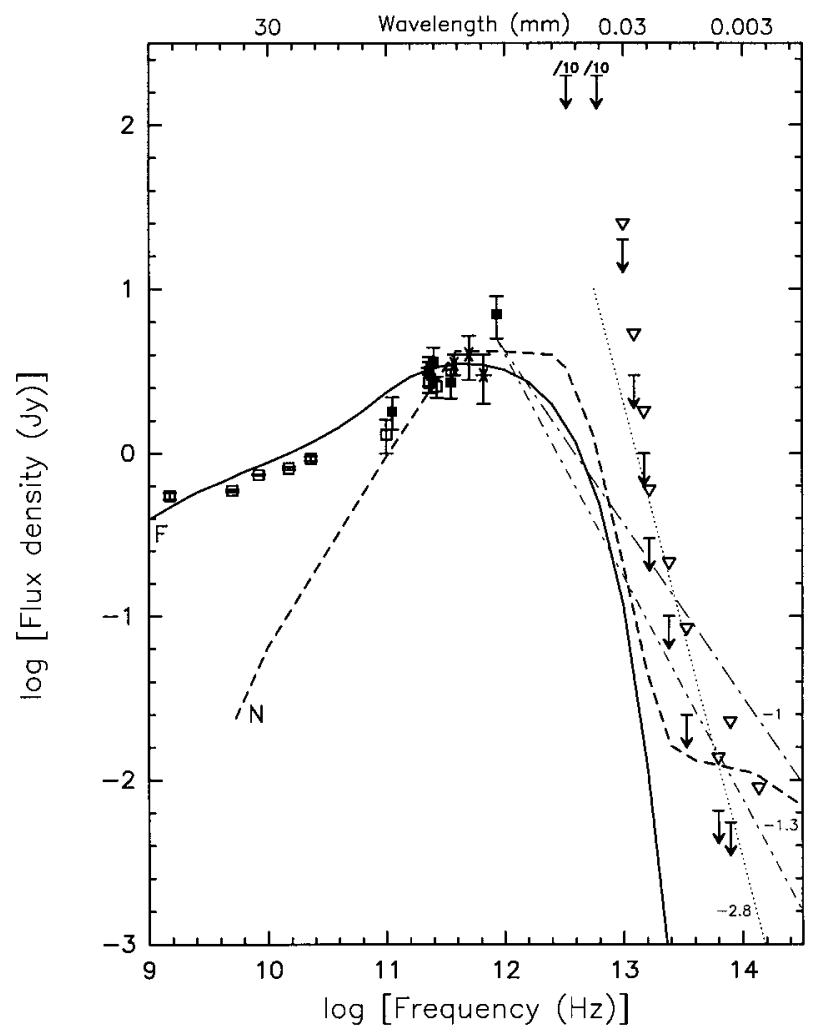

FIG. 4.-Radio-infrared spectrum of Sgr A*. Included are our 1993 (open squares)and 1995/1996 (filled squares) fluxes; the 1994 data of Zy95 (asterisks); FIR upper limits of $2000 \mathrm{Jy}$ at 90 and $50 \mu \mathrm{m}$, estimated from the map of Davidson et al. (1992); the MIR upper limits of Telesco et al. (1996) at 30 $\mu \mathrm{m}$, our own at $24.3 \mu \mathrm{m}$, and those of Gezari (1992) and Gezari et al. (1994) at $20.0,18.1$, and $12.4 \mu \mathrm{m}$; the $8.7 \mu \mathrm{m}$ flux (treated as an upper limit) of Stolovy et al. (1996); and the NIR limits at 4.8 and $3.8 \mu \mathrm{m}$ (Herbst et al. 1993) and at $2.2 \mu \mathrm{m}$ (Menten et al. 1997). The arrows give the observed flux upper limits, while the triangles give the corresponding extinction-corrected limits (according to the curve of Mathis 1990, with $A_{v}=30 \mathrm{mag}$ ). The solid curve (F) shows the model spectrum of Falcke (1996) (leaving out the NIR accretion-disk component), and the dashed curve (N) shows the lowest accretion-rate advection model of Narayan et al. (1995). The long dash-dotted curve is the shallowest power-law falloff $\left(v^{-1}\right)$ consistent with the 350 and 8.7 $\mu \mathrm{m}$ data, the short dash-dotted curve is the shallowest power-law falloff $\left(v^{-1.3}\right)$ consistent with the 350 and $4.8 \mu \mathrm{m}$ data, and the dotted curve is a steep $\operatorname{MIR} v^{-2.8}$ falloff.

Sgr A*'s near-millimeter emission are ruled out by its $230 \mathrm{GHz}$ size of less than $1^{\prime \prime}$, which would call for a dust temperature, $T_{d}$, exceeding $100 \mathrm{~K}$, as well as a steep modified RayleighJeans spectrum. The much flatter spectrum observed might suggest a range of dust temperatures, but the necessary temperatures would still be too low $(3-15 \mathrm{~K})$ to produce the observed flux. Thus, the simplest scenario consistent with Sgr A*'s observed radio-submillimeter spectrum is a source composed of two synchrotron components of different scale sizes. [Note that a separate dust component becoming evident at $\nu \gtrsim 800 \mathrm{GHz}$ cannot yet be ruled out because of the rather weak $\left(<11^{\prime \prime}\right)$ size constraint at $850 \mathrm{GHz}$.]

Numerous infrared data (mostly upper limits) further constrain Sgr A*'s spectrum and are compiled in Figure 4 together with our data and the submillimeter data of $\mathrm{Zy} 95$, which fill the $354-850 \mathrm{GHz}$ gap in our spectrum. The $8.7 \mu \mathrm{m}$ flux from Sgr A*'s general direction (Stolovy, Hayward, \& Herter 1996) is also treated as an upper limit in this figure, because it is not established that this somewhat ridgelike emission feature is associated directly with Sgr A*. The MIR and NIR data, even after correction for $A_{v}=30$ mag according to Mathis (1990), indicate a substantial drop in Sgr A*'s emission by the MIR (Fig. 4). The falloff is also present in luminosity terms, as Sgr A*'s observed $350 \mu \mathrm{m}$ luminosity $\left(\nu L_{\nu} \approx 4.6 \times 10^{35} \mathrm{ergs} \mathrm{s}^{-1}\right)$ exceeds the most recent $2.2 \mu$ m luminosity upper bound (Menten et al. 1997) by at least a factor of 4. This falloff could be due to an intrinsic synchrotron cutoff at FIR to MIR wavelengths, which would imply a magnetic field of $\sim 10 \mathrm{G}$ and electron energies of $\sim 100 \mathrm{MeV}$ (Zy95), but low MIR/NIR fluxes could also simply result from excess extinction local to Sgr A*, a possibility we explore after quantifying Sgr A*'s spectral falloff.

The shallowest FIR/MIR power-law rolloff (for brevity, exponential falloffs will not be pursued here) consistent with our $350 \mu \mathrm{m}$ flux and the extinction-corrected MIR/NIR upper limits arises under the assumption that the $8.7 \mu \mathrm{m}$ emission toward Sgr A* (Stolovy et al. 1996) is indeed associated entirely with Sgr $A^{*}$. This implies an average FIR spectral index, $\alpha_{\mathrm{FIR}}$, of less than -1.0 (Fig. 4). However, a power law this shallow would conflict with the more stringent limits at shorter wavelengths (Fig. 4), especially the $4.8 \mu \mathrm{m}$ upper limit of Herbst, Beckwith, \& Shure (1993). If however the $8.7 \mu \mathrm{m}$ emission is unrelated to $\mathrm{Sgr} \mathrm{A}^{*}$, the most stringent constraint arises from the $4.8 \mu \mathrm{m}$ upper limit, which implies that $\alpha_{\mathrm{FIR}}<-1.3$. Of course, the falloff need not begin at $350 \mu \mathrm{m}$, and raising the spectral turnover frequency, $\nu_{t}$, implies much steeper falloffs. Indeed, for $\nu_{t} \sim 10 \mathrm{THz}, \alpha_{\mathrm{MIR}}<-2.8$. Such a steeply plunging synchrotron spectrum could easily account for low NIR/MIR flux ratios toward Sgr A* (Fig. 4; Stolovy et al. 1996), but such a steep spectrum actually passing through the $8.7 \mu \mathrm{m}$ point would pass uncomfortably closely to a large fraction of the extant MIR upper limits (Fig. 4).

If Sgr A*'s FIR spectral turnover is intrinsic, its synchrotron luminosity is $<10^{3} L_{\odot}$. On the other hand, a plummeting FIR/ MIR spectrum could also result from enhanced extinction along Sgr A*'s line of sight, and for an intrinsically flat or slowly rising synchrotron spectrum extending into the MIR or even the NIR, an additional $A_{\nu}$ of $\sim 100$ mag would be needed. Indeed, the $8.7 \mu \mathrm{m}$ emission toward Sgr A* (Stolovy et al. 1996) provides a candidate for a dusty, extincting region. However, the approximately uniform stellar colors in the central 1" (Eckart et al. 1995) largely restrict any excess extinction near Sgr A* to scales of $\lesssim 10^{3}$ AU.

However, as any extincting region toward Sgr $\mathrm{A}^{*}$ is also subject to the IR emission constraints in Figure 4, such an extincting region must be rather compact. Figure 5 shows the restrictions on source diameter, $\theta$, and $T_{d}$ in the case of azimuthally symmetric, optically thick, single-temperature dust emission. According to Figure 5, the $\theta \lesssim 1000$ AU stellar constraint implies that $T_{d}>110 \mathrm{~K}$, while for the central half-parsec's typical $T_{d}$ of $\approx 150-250 \mathrm{~K}$ (Gezari 1992; Telesco, Davidson, \& Werner 1996), $\theta \lesssim 250$ AU is implied. One possibility for an extincting region is a foreground clump, in which case such a feature's likely transverse motion ( $\left.20 \mathrm{AU} \mathrm{yr}^{-1}\right)$, combined with Sgr A*'s consistent NIR faintness for $\gtrsim 5 \mathrm{yr}$, effectively restricts $\theta$ to $>100$ AU, leaving only the 100-1000 AU range available. However, the high densities implied by the extincting region's compactness $\left(\sim 7 \times 10^{9} / \theta_{\mathrm{AU}} \mathrm{cm}^{-3}\right)$ make it more reasonable to envision a small obscuring region of size $\theta \lesssim 250$ AU (or 30 mas), associated directly with $\mathrm{Sgr} \mathrm{A}^{*}$, perhaps in the form of an outer dusty envelope. Thus, in either case it remains formally possible for extinction to cut off $\mathrm{Sgr}$ A*'s synchrotron spectrum before its radiating electrons do, although the parameter ranges available are fairly restrictive. 


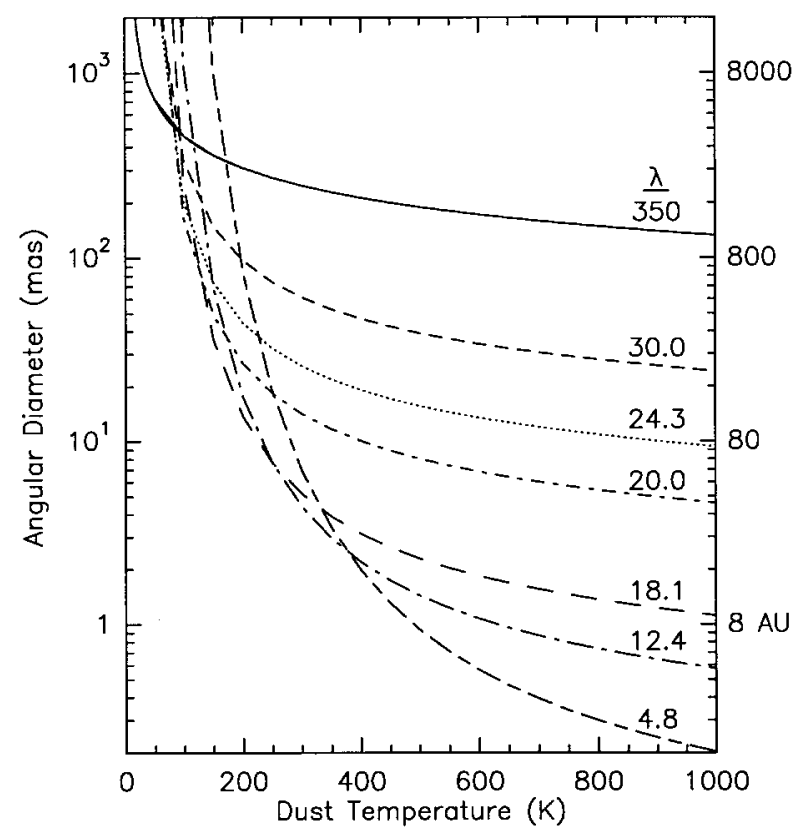

FIG. 5.-Dust temperature source diameter constraints given by the various submillimeter and MIR data. A separate limiting curve is given for each observed wavelength between 350 and $4.8 \mu \mathrm{m}$. The allowed region is to the lower left of all the curves.

MIR cameras on large telescopes will soon enable more sensitive searches for MIR emission from Sgr A*, but another approach to identifying a compact central region of excess extinction would rely on the rapid motions of the stars in the central stellar cusp surrounding Sgr A* (Eckart et al. 1995): any star crossing directly behind a central extincting region should disappear briefly, to reappear on timescales of a few months to several years. It is interesting in this regard that a new NIR source may recently have appeared quite close to Sgr A* (Genzel et al. 1997).

A large extinction very close to Sgr A* would ease concerns regarding Sgr A*'s faintness in the NIR (Menten et al. 1997), but it is not a panacea for accretion-disk hunters, as a large fraction of the central source's purported optical/UV luminosity would then simply emerge via dust reradiation in the MIR/ FIR. However, the narrow frequency window available to any possible FIR emission (Fig. 4) severely restricts the luminosity of such emission: even the most narrowband case of singletemperature dust with a $\nu^{2}$ emissivity law allows an FIR luminosity of no more than $3 \times 10^{4} L_{\odot}$. In the spherically symmetric case, Sgr A*'s optical/UV luminosity is restricted to this level also, as is Sgr A*'s possible MIR/NIR synchrotron emission, thus implying an intrinsic synchrotron cutoff at $\lambda \gtrsim 1 \mu \mathrm{m}$, even in the excess extinction case.

Figure 4 also allows the viability of source models to be addressed. Sgr A*'s dual component spectrum implies a dual component structure, and in the accretion context, disk/halo, disk/jet, and core/envelope morphologies arise (e.g., Narayan, Yi, \& Mahadevan 1995; Falcke 1996; Duschl 1997). With the bulk of Sgr A*'s luminosity emerging in the submillimeter, the primary requirement on models is the generation of a dominant submillimeter synchrotron peak of the correct strength. This requirement is met in the accretion models of both Narayan et al. (1995) and Falcke (1996), in which the submillimeter emission arises in the innermost regions (at several Schwarzschild radii) of an accretion flow onto a massive black hole. Beyond this requirement, Falcke's model also generates appropriate radio power-law emission, with the inclusion of an outflowing jet component. As Figure 5 shows, the resultant spectrum matches Sgr $\mathrm{A}^{*}$ 's entire radio-submillimeter spectrum rather well. However, the presence of a jet remains controversial (Krichbaum et al. 1993; Rogers et al. 1994). With no jet component, the model of Narayan et al. (1995) fails to yield the requisite radio emission without artificially raising the electron temperatures at large radii. On the other hand, in the NIR, the standard accretion-disk emission of Falcke et al. (1993) (not shown in Fig. 4) yields excessive emission even for an accretion rate, $\dot{M}$, as low as $10^{-8.5} M_{\odot} \mathrm{yr}^{-1}$. The stringent NIR bounds on Sgr A* (Fig. 4) thus imply a low $\dot{M}$, radiative efficiency, or both (in the absence of excess local extinction). In this area, Narayan et al. (1995) succeed in predicting very low IR emission even for much higher values of $\dot{M}$ by considering advection of a two-temperature plasma, which yields low radiative efficiencies. Thus, the model ingredients currently most consistent with Sgr A*'s spectrum include submillimeter synchrotron emission from the core of an accretion flow onto a massive black hole, weaker radio emission either from an outflowing jet or a more extended accretion envelope, and only dim optical/ IR emission due to the advection of thermal energy past the event horizon.

Finally, we address Sgr A*'s near-millimeter intensity variations. While the data available are rather limited (see also Zy95), slow (a few months to a year long), low-amplitude variations seem to be the rule. Small near-millimeter fluctuation amplitudes are compatible with fluctuations in $\dot{M}$ (Narayan et al. 1997), but much more rapid variability is expected at small radii (where the high-frequency emission emerges) because of short dynamical and radiative timescales (approximately hours). However, on timescales of hours, Sgr A*'s observed near-millimeter variability is low (Gwinn et al. 1991), and as large and rapid near-millimeter flux changes seem to be rare (see, however, Wright \& Backer 1993), reasonably steady accretion is implied. Strong "variability" might also be inferred if Sgr A* were regularly eclipsed by nearby giant or early-type stars, a possibility only if Sgr A*'s intrinsic size at short wavelengths is near the lower end of its allowed range ( $\sim 0.1-1 \mathrm{AU}$; Morris \& Serabyn 1996). However, the instantaneous eclipse probability for stars in the central 1" stellar cusp near Sgr A* (Eckart et al. 1995), given roughly by the brightness ratio of the central arcsecond $(K \sim 14 \mathrm{~m})$ to that of a single $\mathrm{K}$ giant, is only $\sim 10^{-9}$. (The probability of a stellar orbit sufficiently edge-on to be able to cause an eclipse is larger, $\sim 10^{-3}$ assuming about 10 stars in the volume, but infrequent observation renders the former the more relevant estimate.) As interstellar scintillations are also less important at near-millimeter wavelengths (Zh92), intrinsic fluctuations are thus left, for which the primary candidate remains slow accretion rate variations.

We thank R. Zylka, D. Ward-Thompson, T. G. Phillips, R. Narayan, M. Morris, R. Mahadevan, H. Falcke, and W. J. Duschl for helpful discussions and/or comments on the manuscript. The CSO and OVRO are supported by NSF grants AST96-15025 and AST96-13717. 


\section{REFERENCES}

Davidson, J. A., Werner, M. W., Wu, X., Lester, D. F., Harvey, P. M., Joy, M., \& Morris, M. 1992, ApJ, 387, 189

Dent, W. R. F., Matthews, H. E., Wade, R., \& Duncan, W. D. 1993, ApJ, 410, 650

Duschl, W. J. 1997, in IAU Symp. 184, The Central Regions of the Galaxy and Galaxies, ed. Y. Sofue (Dordrecht: Kluwer), in press

Duschl, W. J., \& Lesch, H. 1994, A\&A, 286, 431

Eckart, A., Genzel, R., Hofmann, R., Sams, B. J., \& Tacconi-Garman, L. E. 1995, ApJ, 445, L23

Falcke, H. 1997, in IAU Symp. 184, The Central Regions of the Galaxy and Galaxies, ed. Y. Sofue (Dordrecht: Kluwer), in press

1996, in IAU Symp. 169, Unsolved Problems of the Milky Way, ed. L Blitz \& P. Teuben (Dordrecht: Kluwer), 169

Falcke, H., Biermann, P. L., Duschl, W. J., \& Mezger, P. G. 1993, A\&A, 270, 102

Genzel, R., Eckart, A., Ott, T., \& Eisenhauer, F. 1997, MNRAS, in press

Gezari, D. 1992, in The Center, Bulge, and Disk of the Milky Way, ed. L Blitz (Dordrecht: Kluwer), 23

Gezari, D., Ozernoy, L., Varosi, F., McCreight, C., \& Joyce, R. 1994, in The Nuclei of Normal Galaxies: Lessons from the Galactic Center, ed. R. Genzel, \& A. I. Harris (Dordrecht: Kluwer), 427

Gwinn, C. R., Danen, R. M., Middleditch, J., Ozernoy, L. M., \& Tran, T. K. 1991, ApJ, 381, L43
Herbst, T. M., Beckwith, S. V. W., \& Shure, M. 1993, ApJ, 411, L21 Krichbaum, T. P., et al. 1993, A\&A, 274, L37

Lo, K. Y. 1994, in The Nuclei of Normal Galaxies: Lessons from the Galactic Center, ed. R. Genzel, \& A. I. Harris (Dordrecht: Kluwer), 395 Mathis, J. S. 1990, ARA\&A, 28, 37

Menten, K. M., Reid, M. J., Eckart, A., \& Genzel, R. 1997, ApJ, 475, L111 Morris, M., \& Serabyn, E. 1996, ARA\&A, 34, 645

Narayan, R., Mahadevan R., Grindlay, J. E., Popham, R, G., \& Gammie, C. 1997, preprint

Narayan, R., Yi, I., \& Mahadevan, R. 1995, Nature, 374, 623

Rogers, A. E. E., et al. 1994, ApJ, 434, L59

Serabyn, E., Carlstrom, J. E., \& Scoville, N. Z. 1992, ApJ, 401, L87

Stolovy, S., Hayward, T., \& Herter, T. 1996, ApJ, 470, L45

Telesco, C. M., Davidson, J. A., \& Werner M. W. 1996, ApJ, 456, 541

Wang, N., et al. 1996, Appl. Opt., 35, 6629

Wright, M. C. H., \& Backer, D. C. 1993, ApJ, 417, 560

Zhao, J.-H., Goss, W. M., Lo, K. Y., \& Ekers, R. D. 1992, in Relationships between Active Galatic Nuclei and Starburst Galaxies, ed. A. V. Filipenko (San Francisco: ASP), 295 (Zh92)

Zylka, R., Mezger, P. G., \& Lesch, H. 1992, A\&A 261, 119 (Zy 92)

Zylka, R., Mezger, P. G., Ward-Thompson, D., Duschl, W. J., \& Lesch, H. 1995, A\&A, 297, 83 (Zy95) 


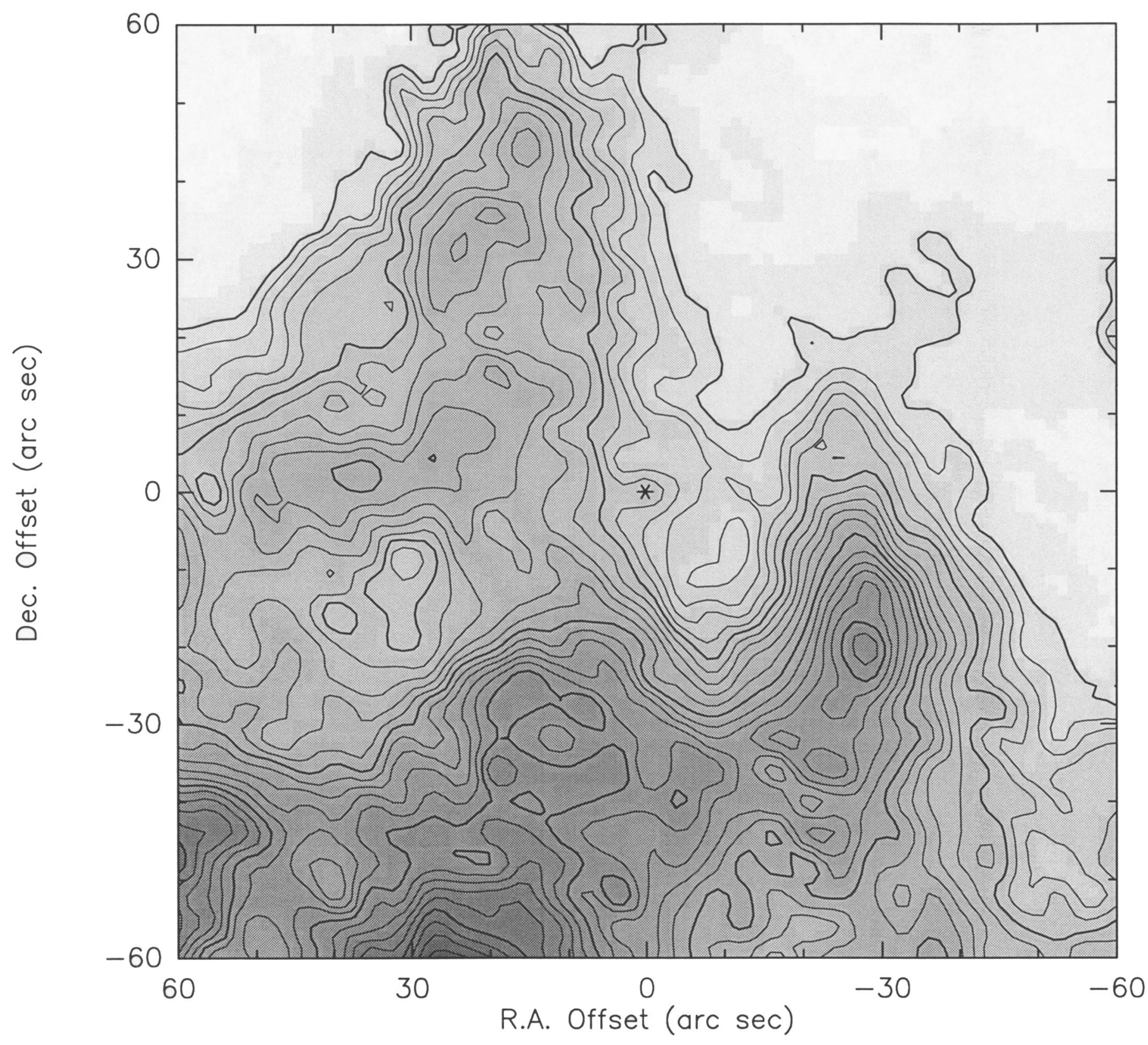

FIG. 3.-11" resolution SHARC map of the vicinity of Sgr $\mathrm{A}^{*}$ at $\lambda=350 \mu \mathrm{m}$. This image is the central section of a much larger map, acquired by scanning the linear SHARC array in azimuth, while chopping $89^{\prime \prime}$ in azimuth. The pointing of this map was corrected by $9^{\prime \prime}$ in R.A. by correlating its prominent large-scale dust emission features with the $450 \mu \mathrm{m}$ maps of Dent et al. (1993) and Zy95. The resultant position of Sgr A* is marked with an asterisk. (The familiar western arc feature peaks at $\left(-28^{\prime \prime},-20^{\prime \prime}\right)$, while the northern arm extends to the top of the map from northeast of Sgr $\mathrm{A}^{*}$.) The contour levels are $36,39,42, \ldots$. Jy beam $^{-1}$.

SERABYN et al. (see 490, L77) 